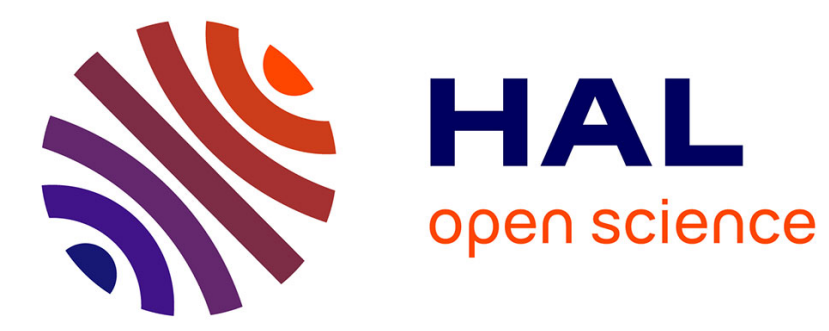

\title{
Inciter à différer le départ en retraite: une analyse en termes de courbe de Laffer
}

Jean-Olivier Hairault, François Langot, Thepthida Sopraseuth

\section{To cite this version:}

Jean-Olivier Hairault, François Langot, Thepthida Sopraseuth. Inciter à différer le départ en retraite: une analyse en termes de courbe de Laffer. Revue d'Economie Politique, 2005, 115, pp.241-263. halshs-00270278

\section{HAL Id: halshs-00270278 \\ https://shs.hal.science/halshs-00270278}

Submitted on 4 Apr 2008

HAL is a multi-disciplinary open access archive for the deposit and dissemination of scientific research documents, whether they are published or not. The documents may come from teaching and research institutions in France or abroad, or from public or private research centers.
L'archive ouverte pluridisciplinaire HAL, est destinée au dépôt et à la diffusion de documents scientifiques de niveau recherche, publiés ou non, émanant des établissements d'enseignement et de recherche français ou étrangers, des laboratoires publics ou privés. 


\title{
Inciter à différer le départ en retraite : une analyse en termes de courbe de Laffer
}

\author{
Jean-Olivier Hairault * $\quad$ François Langot ${ }^{\dagger} \quad$ Thepthida Sopraseuth ${ }^{\ddagger}$
}

Décembre 2004

\begin{abstract}
Résumé
En France, une année supplémentaire au-delà du taux plein dans le régime général ne donne droit à aucune augmentation de pension. Cette taxe sur le prolongement d'activité distort les choix des agents en faveur d'un âge de départ en retraite inférieur à celui qui prévaudrait dans un contexte optimal. Cette taxe pourrait être éliminée par la mise en place d'un système qui lierait le taux de remplacement à l'âge de départ en retraite de façon à respecter la neutralité actuarielle. Pour des raisons exactement opposées à la situation prévalant actuellement, ce système ne permet pas d'assurer des moyens de financement supplémentaires pour les caisses des régimes par répartition. Il existe en effet un dilemme entre une taxe élevée et un allongement significatif de la durée d'activité. Nous présentons un modèle très simplifié à deux périodes qui permet de déterminer de façon explicite et transparente la taxe sur la prolongation d'activité qui correspond au sommet de la courbe de Laffer. Dans un second temps, nous présentons, sur la base d'une maquette beaucoup plus détaillée de l'économie française, des résultats quantitatifs qui illustrent de façon concrète notre analyse théorique.
\end{abstract}

${ }^{*}$ EUREQua, Université Paris I et CEPREMAP. Adresse : EUREQua, 106-112 Bd de l'Hôpital, 75447 Paris Cedex 13. Email : joh@univ-paris1.fr

${ }^{\dagger}$ GAINS, CEPREMAP et Université du Maine. Adresse : Faculté de Droit et des Sciences Économiques, Université du Maine, Avenue O. Messiaen, 72085 Le Mans Cedex 9 Email : flangot@univ-lemans.fr

${ }^{\ddagger}$ EPEE, CEPREMAP et Université d'Evry Val d'Essonne. Adresse : Université d'Evry Val d'Essonne, Département d'Economie, 4 Bd F. Mitterand, 91025 Evry Cedex. Email : tsoprase@univ-evry.fr

${ }^{\S}$ Ce travail a été financé par le Commissariat Général au Plan, dans le cadre d'un contrat avec le Cepremap, finalisé en décembre 2002. Nous avons bénéficié de discussions avec P.Y. Hénin et T. Weitzenblum dans le cadre de ce projet de recherche. Nous les en remercions chaleureusement, ainsi que P. Artus, L. Caussat, T. Japelli, G. Laroque, A. Lavigne, F. Legros, M. Pagano, P. Pestieau, B. Sédillot et E. Walraet. Nous remercions également pour leurs commentaires les participants à la journée scientifique de la Caisse des Dépôts et Consignations (Bordeaux, 2003), le 1er workshop "Transferts inter-générationnels et marchés financiers" (Paris, 2003), l'Association Française des Sciences Economiques (Paris, 2003), T2M (Orléans, 2004), le séminaire Fourgeaud (Paris, 2003), le congrès de l'EEA Congress (Stockholm, 2003), la Society for Computational Economics (Seattle, 2003), le CSEF (Naples, 2003) et la SED (Paris, 2003). Nous restons seuls responsables des insuffisances et erreurs qui demeureraient dans ce travail. 


\section{Introduction}

Les régimes de retraite par répartition pèsent fortement sur les décisions de départ à la retraite ([Gruber \& Wise, 1998]). Ainsi, en France, une année supplémentaire au-delà du taux plein dans le régime général ne donne droit à aucune augmentation de pension ${ }^{1}$. Pourtant, elle implique des cotisations supplémentaires (taxe explicite) et une année en moins de versement de la pension (taxe implicite). C'est pourquoi il est courant de parler de taxe sur la prolongation d'activité ([Desmet \& Pestieau, 2003]). Elle distort les choix des agents en faveur d'un âge de départ en retraite inférieur à celui qui prévaudrait dans un contexte d'optimalité. Cette taxe pourrait être éliminée par la mise en place d'un système qui lierait le taux de remplacement à l'âge de départ en retraite, système qualifié traditionnellement d'actuariellement neutre : l'augmentation de la pension suite à une année supplémentaire d'activité compense exactement les cotisations et le manque à gagner en termes d'années de retraite. Si ce système permet de corriger une distorsion dans l'allocation des ressources, il ne permet pas, par définition, d'assurer des sources de financement pour les caisses des régimes par répartition. De ce point de vue, il est strictement équivalent à la situation actuelle, mais pour des raisons exactement opposées : il n'existe pas de recettes supplémentaires, produit d'une taxe sur des années de prolongation d'activité, en raison, soit d'une taxe qui s'annule dans le cas d'une réforme instaurant la neutralité actuarielle, soit d'une "assiette" inexistante si l'on considère le système actuel. Il s'agit là des deux points extrêmes de la courbe de Laffer sur la taxe à la prolongation d'activité. Il semble en effet éclairant de présenter les enjeux financiers sur le report de l'âge de la retraite en termes de courbe de Laffer. Si l'Etat prélève une part importante des surplus engendrés par les années de prolongation d'activité, en proposant une surcote très en deçà de la surcote actuariellement neutre, il prend le risque de dissuader ce type de comportement, tandis qu'il n'y aurait aucun rendement à repousser fortement l'âge de la retraite grâce à des surcotes actuariellement neutres. La question centrale sous l'angle des finances publiques est de déterminer la surcote correspondant au sommet de la courbe de Laffer.

Dans une première section, nous présentons un modèle très simplifié à deux périodes qui permet de déterminer de façon explicite et transparente la taxe sur la prolongation d'activité qui correspond au sommet de la courbe de Laffer. Le modèle reprend les formulations utilisées dans la littérature pour analyser les choix de départ en retraite ([Artus \& Legros, 1999], [D’Autume, 2003], [Desmet \& Pestieau, 2003]). La préférence relative pour le loisir et l'horizon de vie apparaissent alors comme les facteurs-clés de cette analyse. Dans une seconde section, nous exposons, sur la base d'une maquette beaucoup plus détaillée de l'économie française, déjà présentée dans [Hairault et al., 2004], des résultats quantitatifs qui illustrent de façon concrète notre analyse théorique. Nous donnons en fonction du paramètre de préférence pour le loisir un intervalle de confiance des gains financiers (permettant de réduire les déficits futurs) que la CNAV pourrait engendrer par une mise en place de surcotes. La situation actuelle nous fournit un niveau de préférence pour le loisir minimal en deçà duquel les agents choisissent de reporter leur âge de départ au-delà du taux plein. Comme cette situation est en contradiction avec l'observation, il s'agit bien d'un seuil en dessous duquel on ne peut descendre pour calibrer ce paramètre structurel. Nous comparons alors deux scénarios, l'un prenant cette valeur seuil qui est la plus favorable des hypothèses pour la réforme envisagée, l'autre reposant sur une calibration macroéconomique visant à reproduire au niveau agrégé le ratio consomma-

\footnotetext{
${ }^{1}$ Ceci était vrai avant la réforme du gouvernement Raffarin qui institue dorénavant une majoration de $3 \%$ par année de report.
} 
tion/produit. L'apport de cet article par rapport aux conclusions de [Hairault et al., 2004] réside dans la mesure de la sensibilité des résultats à la préférence pour le loisir, ainsi que l'explicitation de la courbe de Laffer dans le cadre d'un modèle à 2 périodes. Nous évoquons en conclusion les problèmes qui hypothèquent encore ce type de réformes.

\section{Un modèle analytique à 2 périodes}

Considérons un modèle à générations à 2 périodes de vie, sans altruisme. En deuxième période de vie de longueur déterministe $T<1$, en plus de leur consommation $d$, les agents décident de leur âge de retraite $z$. On suppose pour simplifier qu'il n'existe aucune source de croissance, ni démographique, ni technologique. Le système de retraite par répartition prélève une fraction $\theta$ du salaire (de la productivité du travail) $w$, les agents offrant de façon inélastique une quantité de travail normalisée à 1 .

\subsection{Un régime à prestations définies}

Le taux de remplacement à la retraite est égale à $\lambda$, quelle que soit la durée d'activité en deuxième période. Tous les agents ont accès au même taux de remplacement au-delà d'une durée minimale de cotisation qui correspond ici à la fin de la période 1. L'équilibre des caisses de retraite implique le taux de remplacement suivant :

$$
\lambda=\frac{\theta(1+z)}{T-z}
$$

Chaque ménage maximise son utilité intertemporelle $U$ (fonction croissante dans les consommations et décroissante dans l'âge de retraite) :

$$
\max _{c \geq 0, d \geq 0, z \geq 0} U(c, d, z)
$$

sous les contraintes budgétaires de première et seconde période :

$$
\begin{aligned}
c & =(1-\theta) w \\
d & =(1-\theta) z w+\lambda w(T-z)
\end{aligned}
$$

Dans ce modèle simplifié à 2 périodes, nous faisons abstraction de l'épargne pour mettre l'accent sur les choix de départ dans le cadre du système de retraite par répartition. La maximisation de l'utilité intertemporelle sous les contraintes (2) et (3) conduit à la condition suivante concernant le taux marginal de substitution entre $d$ et $z$ :

$$
\frac{U_{z}^{\prime}}{U_{d}^{\prime}}=-(1-\theta-\lambda) w
$$

Le système de retraite par répartition via $\theta$ et $\lambda$ influence la date de retraite $z$ qui diffère alors du TMS du planificateur ${ }^{2} z^{*}$ définie par :

$$
\frac{U_{z^{*}}^{\prime}}{U_{d^{*}}^{\prime}}=-w
$$

\footnotetext{
${ }^{2}$ Nous ne cherchons pas à optimiser la valeur de $\theta$ que nous considérons comme donnée. Nous ne discutons que de l'optimalité entre date de départ à la retraite et consommation de deuxième période dans le cadre d'un arbitrage intertemporel déterminé par la valeur donnée du taux de cotisation.
} 
$(\theta+\lambda)$ constitue une taxe à la prolongation d'activité, explicite via le taux de cotisation, mais également implicitement à cause de la réduction du temps passé à recevoir la pension de retraite. L'existence de cette taxe implique un âge de départ à la retraite plus faible qu'à l'optimum contraint (à $\theta$ donné), à la condition naturellement que l'effet substitution domine dans le cas où $z^{*}>0$.

\subsection{Les surcotes actuariellement neutres}

Il est possible de restaurer le taux marginal de substitution de premier rang entre la consommation de deuxième période et l'âge de départ à la retraite en modifiant le système de calcul des pensions, plus précisément en introduisant une indexation de la pension sur la durée d'activité, et ceci de façon actuariellement neutre. Le ratio de remplacement $\lambda_{1}$ détermine uniquement le niveau de retraite minimal auquel les ménages ont droit à la fin de la période 1 . Tout prolongement d'activité dans la période 2 augmente les droits à la retraite. Le ratio de remplacement est alors la somme de deux composantes :

$$
\lambda=\lambda_{1}+\lambda_{2}^{*}(z)
$$

Le ratio de remplacement en cas de départ en retraite en fin de première période, $\lambda_{1}$, est déterminé par l'équilibre du système de retraite par répartition pour ce choix de départ à la retraite :

$$
\lambda_{1}=\frac{\theta}{T}
$$

Sachant (1), (5) et (6), le ratio de remplacement spécifique au départ en retraite différé s'écrit :

$$
\lambda_{2}^{*}(z)=\frac{z\left(\theta+\lambda_{1}\right)}{T-z}
$$

Ce bonus ou surcote actuariellement neutre permet de compenser les cotisations et les années de non-versements de pensions. Dans ce cas, la contrainte budgétaire de deuxième période se réécrit :

$$
d=z w+\lambda_{1} w T
$$

On retrouve alors le taux marginal de substitution du planificateur et par définition les caisses de retraite sont équilibrées.

\subsection{La mise en évidence d'une courbe de Laffer}

L'idée dans ce papier est d'introduire un bonus $\lambda_{2}$ croissant avec $z$ mais qui ne représente qu'une fraction ${ }^{3}(1-\tau)$ de la surcote actuariellement neutre afin de maximiser l'excédent des caisses de retraite :

$$
\lambda_{2}(z)=(1-\tau) \lambda_{2}^{*}(z)
$$

La contrainte budgétaire de seconde période devient dans ce cas :

$$
d=\left(1-\tau\left(\theta+\lambda_{1}\right)\right) w z+\lambda_{1} w T
$$

L'arbitrage entre consommation de seconde période et loisir est donc résumé par le TMS suivant :

$$
\frac{U_{z}^{\prime}}{U_{d}^{\prime}}=-\left(1-\tau\left(\theta+\lambda_{1}\right)\right) w
$$

\footnotetext{
${ }^{3}$ Nous nous restreignons à une fraction constante pour simplifier l'analyse.
} 


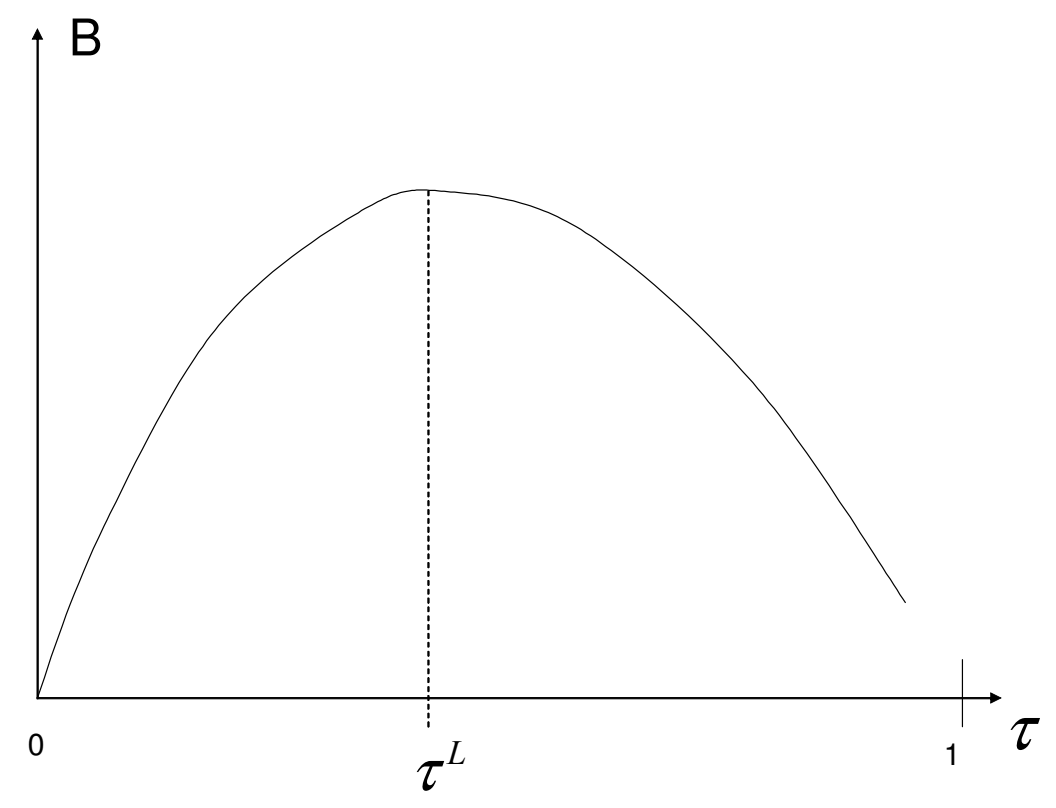

FIG. 1 -

Nous vérifions que nous retrouvons le TMS du planificateur pour $\tau=0$. On peut alors écrire l'excédent budgétaire $B$ de la façon suivante :

$$
B=\theta(z+1) w-\left(\lambda_{1}+\lambda_{2}\right) w(T-z)
$$

Sachant (6), (7) et (8), l'expression de l'excédent budgétaire devient

$$
B=\left(\theta+\lambda_{1}\right) z \tau w
$$

Lorsque les individus reçoivent les incitations actuariellement neutres $(\tau=0)$, par définition, le surplus est nul $(B=0)$. Des valeurs croissantes de $\tau$ améliorent l'excédent budgétaire à $z$ donné. Toutefois, les individus étant moins incités à travailler ${ }^{4}$, la durée de prolongation $z$ s'éloigne de l'optimum $z^{*}$. La courbe de Laffer résulte de ces deux effets contrastés de $\tau$ sur l'excédent budgétaire. Il existe donc un niveau de taxe sur le travail, ou de manière équivalente, un niveau $\tau^{L}$ qui maximise l'excédent budgétaire (graphique 1). Au sommet de la courbe de Laffer $\left(\frac{\partial B}{\partial \tau}=0\right)$, l'élasticité de $z$ par rapport à $\tau$ est unitaire :

$$
\frac{\partial z}{\partial \tau} \frac{\tau}{z}=1
$$

\subsection{Une détermination explicite}

La prise en compte d'une fonction d'utilité explicite et simple permet d'éclairer notre raisonnement. Considérons la fonction d'utilité suivante ([D'Autume, 2003]) :

$U=\log c+\beta T \log \left(\left(\frac{d}{T}\right)^{1-m}\left(\frac{T-z}{T}\right)^{m}\right)=\log c+\beta T[((1-m) \log d+m \log (T-z)-\log T)$

\footnotetext{
${ }^{4}$ Sous l'hypothèse naturellement que $z$ soit une fonction décroissante de $\tau$, ce qui est vérifié par exemple pour une fonction d'utilité instantanée logarithmique.
} 
Dans ce cas, l'équation (10) conduit au TMS suivant :

$$
\frac{d}{T-z} \frac{m}{1-m}=\left[1-\tau\left(\theta+\lambda_{1}\right)\right] w
$$

Sachant (9), la décision de départ est égale à :

$$
z=(1-m) T-\frac{\lambda_{1} T m}{1-\tau\left(\theta+\lambda_{1}\right)}
$$

L'équation (12) confirme que l'individu réduit son activité lorsque la taxe implicite sur le travail $\tau\left(\theta+\lambda_{1}\right)$ augmente. L'âge de départ est strictement inférieur à l'âge obtenu dans le cas optimal $\tau=0$ dès lors que $z^{*}>0$, ce qui est vrai à la condition que

$$
\frac{m}{1-m}<\frac{T}{\theta}
$$

La préférence pour le loisir $m$ a une influence négative sur $z$, contrairement à la longueur $T$ de la période 2 qui s'interprète comme l'horizon de vie.

En reportant cette expression dans le surplus (equation (11)), nous déterminons le niveau de la taxe $\tau^{L}$ qui maximise l'excédent budgétaire :

$$
\tau^{L}=\frac{1-\sqrt{\frac{\frac{\theta}{T} m}{1-m}}}{\theta\left(1+\frac{1}{T}\right)}
$$

Plus la préférence pour le loisir est faible, plus la taxe sur le prolongement d'activité doit rester élevée. Un horizon de vie $T$ important joue un rôle similaire. Ces deux facteurs vont en effet dans le sens d'un âge de la retraite retardé ( $z$ élevé). Compte tenu de (14), le choix de départ en retraite correspondant au maximum de la courbe de Laffer est donné par l'expression suivante :

$$
z^{L}=(1-m) T-\frac{\lambda_{1} T m}{\sqrt{\frac{\lambda_{1} m}{1-m}}}=(1-m) T-\frac{\theta m}{\sqrt{\frac{\theta m}{T(1-m)}}}
$$

\subsection{Retraite choisie et réduction des déficits}

L'allongement de la durée de la vie crée indubitablement des tensions sur le régime de retraite par répartition. Un déficit apparaît de façon inéluctable lorsque le taux de cotisation et le ratio de remplacement demeurent inchangés. Dans quelle mesure l'introduction de surcotes permet-elle de réduire les déficits attendus?

Considérons un allongement de la durée de vie jusqu'à $T_{2}>T_{1}$. L'excédent budgétaire s'écrit alors

$$
B=\theta(z+1) w-\left(\lambda_{1}+\lambda_{2}\right) w\left(T_{2}-z\right)
$$

Si les caisses de retraite souhaitent préserver la générosité du système, le ratio de remplacement en cas de départ en retraite dès la fin de la première période demeure inchangé : $\lambda_{1}=\frac{\theta}{T_{1}}$. En revanche, on a une modification de la définition des surcotes :

$$
\lambda_{2}=(1-\tau) \frac{z\left(\theta+\lambda_{1}\right)}{T_{2}-z}
$$


En reportant ces expressions dans (15), l'excédent budgétaire s'écrit

$$
B=\theta\left[1-\frac{T_{2}}{T_{1}}\right] w+\left(\theta+\lambda_{1}\right) z \tau w
$$

Le premier terme du membre de droite de l'équation (16) fait apparaître le déficit budgétaire associé à l'allongement de l'horizon de vie, sachant qu'en cas de départ à l'âge minimal (en fin de période 1), la générosité du système de retraite demeure inchangée $\left(\lambda_{1}=\frac{\theta}{T_{1}}\right)$. La seconde composante $\left(\theta+\lambda_{1}\right) z \tau w$, déjà présente dans (11), constitue une source de financement de ce déficit, en incitant les individus à travailler davantage par le biais d'incitations actuariellement neutres sur lesquelles les caisses de retraite prélèvent une fraction $\tau$. Nous retrouvons naturellement la même condition définissant le sommet de la courbe de Laffer (équation (14)), niveau de la taxe qui maximise les surplus budgétaires pouvant être engendrés par la prolongation d'activité et susceptibles de financer les déficits occasionnés par l'allongement de la durée de vie.

Cependant, il faut noter que l'existence d'un déficit budgétaire non couvert implique, toutes choses égales par ailleurs, par un effet richesse, que la durée de la vie active est plus courte, ie. $z$ est plus faible. En particulier, lorsque $\tau=0$, on ne retrouve plus $z^{*}$, mais une valeur inférieure :

$$
z_{\tau=0}=T_{2}(1-m)-\theta m \frac{T_{2}}{T_{1}}=z^{*}-\theta m\left(\frac{T_{2}}{T_{1}}-1\right)
$$

Pour que la politique d'incitations ait une certaine efficacité, il est nécessaire que $z_{\tau=0}$ soit positif. Cela implique la condition suivante :

$$
\frac{m}{1-m}<\frac{T_{1}}{\theta}
$$

La condition (17) est plus restrictive que la condition (13) qui s'applique pour $T=T_{2}$.

\subsection{Une illustration empirique}

La section 3 fournit une évaluation quantitative de la courbe de Laffer sur données françaises et cherche à déterminer la fraction de la surcote actuariellement neutre que les caisses de retraite doivent prélever pour financer le déficit inhérent à un allongement de l'horizon de vie. Ici, nous illustrons sur la base très simplifiée du modèle à deux périodes cette analyse quantitative.

Considérons que les périodes du modèle correspondent à 40 années. Le ratio de remplacement $\lambda_{1}$ de référence est calculé pour un horizon de vie de 20 ans et pour un taux de cotisation de $25 \%$. Pour partir d'une situation réaliste, le paramètre de désutilité du travail $m$ est fixé de façon à être compatible avec les décisions de départ en retraite observés aujourd'hui. Actuellement, les caisses de retraite ne fournissent pas d'incitations à travailler au-delà de leur taux plein $(\tau=1)$, ce qui conduit les individus, dont la carrière est complète, à partir en retraite à l'âge de 60 ans $(z=0)$ : les préférences des agents et le système de retraite conduiraient spontanément à $z \leq 0$. L'équation (12) avec $\tau=1$ permet alors de définir les valeurs de $m$ compatible avec l'observation :

$$
\frac{m}{1-m} \geq \frac{(1-\theta) T_{1}-\theta}{\theta}
$$


TAB. 1 - Etalonnage du modèle analytique

\begin{tabular}{llc}
\hline \hline Paramètre & & Valeur \\
\hline Espérance de vie initiale & $T_{1}:$ & $\frac{20}{40}$ \\
Espérance de vie allongée & $T_{2}:$ & $\frac{26}{40}$ \\
Taux de cotisation & $\theta:$ & 0.25 \\
Salaire & $w:$ & 1 \\
Utilité du loisir minimale & $\underline{m}:$ & 0.33 \\
Utilité du loisir maximale & $\bar{m}:$ & 0.66 \\
\hline \hline
\end{tabular}

La condition (18) définit implicitement un niveau de l'utilité du loisir $\underline{m}$ en dessous duquel les agents décaleraient leur départ à la retraite au-delà de leur taux plein. Nous ne considérerons donc que des valeurs supérieures à $\underline{m}$. Or, la condition (17) définit une valeur maximale $\bar{m}$ de l'utilité du loisir au-delà de laquelle les politiques incitatives sont inefficaces. Nous restreignons par la suite l'analyse de notre politique à cet intervalle $[\underline{m}, \bar{m}]$.

Supposons que l'espérance de vie augmente de 6 ans, ce qui est prévu à l'horizon 2040. Sur la base de ce modèle simplifié, un allongement de $T_{1}$ à $T_{2}$ de l'horizon de vie implique que $\left(1-T_{1} / T_{2}\right) \%$ des pensions ne sont pas financées lorsque le système n'est pas modifié. Dans le scénario présenté, cela impliquerait que $23 \%$ des pensions ne seraient pas financées, ce qui, compte tenu de la non-prise en compte des problèmes liés à l'effet baby-boom, est assez réaliste. Quelle fraction des surcotes actuariellement neutres permet alors de réduire au maximum le déficit des caisses de retraite?

Le graphique 2 traduit la réduction du déficit (en \%) induite par des incitations dont l'ampleur dépend de $\tau$. Ce calcul est effectué pour 3 étalonnages intermédiaires de l'utilité du loisir. Dans les 3 cas apparaît une courbe de Laffer : lorsque les incitations sont fortes $(\tau$ proche de 0$)$, les individus retardent leur départ en retraite mais se rapprocher de la neutralité actuarielle rend le budget des caisses de retraite peu sensible à cet allongement de la durée d'activité. Symétriquement, lorsque les incitations sont faibles ( $\tau$ proche de 1), les individus retardent peu leur départ en retraite, ce qui n'améliore pas la situation financière des caisses de retraite. Il existe, pour chaque valeur de $m$, un taux $\tau^{L}$ qui maximise la réduction du déficit.

L'ampleur de la réduction du déficit pour le niveau optimal $\tau^{L}$ s'avère très sensible à l'utilité du loisir. Lorsque les individus valorisent beaucoup le loisir, les caisses de retraite ne peuvent prélever qu'une part $\tau^{L}$ faible des incitations actuariellement neutres et la réduction du déficit qui lui est associée décline. Ainsi, pour une utilité du loisir faible $m=0.4$, le sommet de la courbe de Laffer correspond à une réduction très forte du déficit occasionné par un allongement de 6 ans de la durée de vie. A titre indicatif, cela entraîne une augmentation de la durée d'activité au-delà du taux plein de 6,5 années. Une valeur plus élevée, par exemple $m=0.567$, conduit à une réduction plus modique, de l'ordre de $10 \%$, et à un allongment de la durée d'activité de seulement 2 ans. Ce dernier cas est assez similaire aux résultats obtenus dans le cadre de notre modélisation plus réaliste présentée dans la section suivante.

\section{Une évaluation quantitative de la courbe de Laffer}

La section précédente permettait de présenter de façon explicite l'argument de la courbe de Laffer sur la taxe à la prolongation d'activité. Dans un article récent ([Hairault et al., 2004]), 
Fig. 2 - Courbes de Laffer pour des valeurs croissantes de l'utilité du loisir

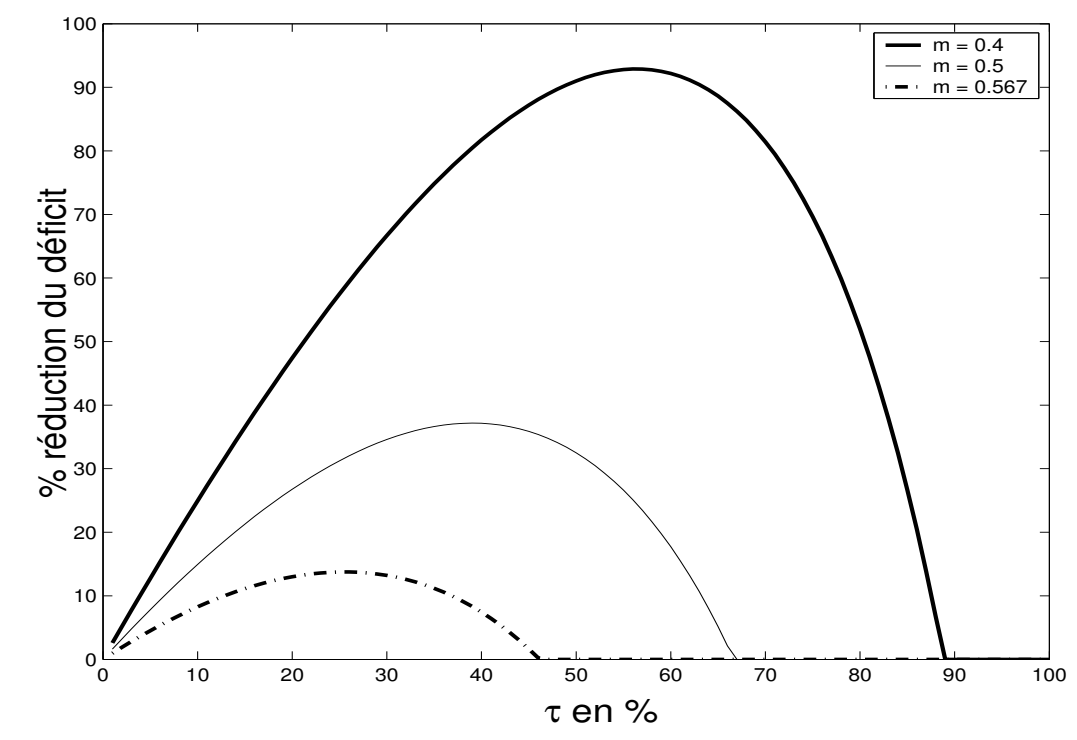

nous avons tenté de déterminer précisément le sommet de la courbe de Laffer dans le cadre du régime par répartition existant en France dans le secteur privé. C'est parce qu'actuellement la taxe sur l'activité est particulièrement forte que ce type de mesure est susceptible de fournir des moyens de financer les déficits attendus du régime par répartition. Cette analyse quantitative implique cependant d'inclure toute une série d'éléments susceptibles d'influer sur les décisions de départ en retraite.

\subsection{Eléments de la modélisation}

Pour analyser l'incidence de la législation sur les droits de retraite et surtout celle des incitations qui peuvent être mises en place, nous proposons un modèle de cycle de vie où les décisions de départ à la retraite sont naturellement endogènes. L'originalité de notre approche est d'intégrer ces décisions de participation dans le cadre d'un modèle à générations imbriquées, explicitant les choix endogènes de consommation et d'épargne. En effet, l'interaction entre les choix de départ en retraite et les choix d'épargne peuvent être essentiels : des montants élevés de patrimoine financier peuvent permettre à certains agents de partir plus tôt en retraite pour profiter du loisir. Dans cette étude, nous optons pour un type de modèle à génération où les principales étapes de la vie sont représentées par des variables aléatoires. Cette stratégie de modélisation constitue une autre originalité de ce travail, et généralise les travaux de [Yaari, 1965], [Blanchard, 1985], [Gertler, 1999] ou [Castañeda et al., 2002] en reprenant ceux de [Hairault \& Langot, 2002]. Elle permet de fonder le lien entre les générations se succédant dans le temps sur la base de la valeur du bien-être des enfants : ainsi, les choix optimaux d'épargne sont conditionnés par l'anticipation des besoins d'épargne des descendants ${ }^{5}$. Comme la carrière salariale des enfants est incertaine, les parents prudents épargnent alors pour leur léguer un patrimoine. Ainsi, l'épargne de précaution est ici un transfert inter-générationnel. Nous introduisons une contrainte de liquidités synonyme d'imperfections sur les marchés financiers : il est im-

\footnotetext{
${ }^{5}$ De plus, avec ce type de préférence, il est possible de moduler le degré d'altruisme.
} 
possible de s'endetter, ce qui permet, de façon simplifiée, de rendre compte de l'existence de contraintes financières. De plus, nous supposons qu'il n'existe pas d'actifs financiers assurant des rentes viagères. Le seul actif financier disponible est un actif rapportant un intérêt certain.

En outre, cette stratégie de modélisation permet de prendre en compte de nombreuses sources d'hétérogénéité. Au début de leur vie active, les agents ne connaissent pas avec certitude leur date de départ en retraite, leur date de promotion et leur date de mort. Cette représentation markovienne de l'âge permet, de par sa parcimonie, d'introduire des choix endogènes de départ en retraite conditionnés d'une part par le niveau d'actifs financiers des agents mais également par les droits de retraite accumulés tout au long de leurs carrières professionnelles. En effet, notre modélisation intègre une représentation fine du régime général et des deux principaux régimes de retraite complémentaire (ARRCO et AGIRC). De plus, aujourd'hui, en France, seul un retraité sur deux est salarié avant de prendre sa retraite. La baisse du taux d'activité des travailleurs âgés constitue un élément essentiel dans le choix de départ en retraite : puisque chômeurs et salariés disposent de revenus courants et d'une retraite espérée différente, une analyse des décisions de départ en retraite doit tenir compte de cette dégradation du taux de participation des travailleurs âgés. Le modèle tient effectivement compte des risques de chômage et de pré-retraite en fin de vie active.

Contrairement aux travaux de l'équipe DESTINIE ([Bardaji et al., 2002]), notre modélisation intègre des fondements micro-économiques permettant d'effectuer des variantes de politique économique. Notre modèle permet également de "dépasser" les analyses menées par [Blanchet \& Mahieu, 2001], [Mahieu \& Sédillot, 2001], [Rust \& Phelan, 1997] ou [INGENUE, 2001] par exemple, car la distribution de la richesse financière des agents et les choix de départ en retraite deviennent endogènes. Cette méthodologie doit alors permettre d'apprécier les réactions des différents agents aux modifications des règles institutionnelles ou aux incitations en endogénéisant les distributions des départs à la retraite et celle des richesses. L'accumulation d'actif financier affecte en particulier les décisions de départ en retraite par deux effets contradictoires. D'une part, les individus riches peuvent partir en retraite plus tôt, substituant aux revenus de la pension des revenus financiers. D'autre part, le désir d'accumuler un patrimoine financier incite certains individus à différer leur départ en retraite. Cet effet est d'autant plus marqué que la pension de retraite récompense l'activité prolongée ([Hairault et al., 2004]).

Nous ne reviendrons pas ici sur la description détaillée du modèle présentée dans [Hairault et al., 2004]. Rappelons que ce modèle de cycle de vie comprend 3 catégories socio-professionnelles (CSP) : les cadres, les professions intermédiaires et les employés - ouvriers. L'étalonnage du modèle tient compte des spécificités de chaque CSP en ce qui concerne leurs espérances de vie, leurs parcours professionels (âge d'entrée dans la vie active, risque de chômage, salaire au long de la vie) et le calcul de leurs pensions de retraite (ARRCO pour tous les salariés et AGIRC pour les cadres). L'étalonnage spécifique à chaque CSP nous permet d'intégrer dans le modèle le calcul de la pension de retraite. Les choix endogènes de participation au marché du travail après 59 ans rend endogène le ratio de remplacement à la retraite. Nous tenons enfin compte de la mobilité sociale intergénérationnelle, étalonnée sur les données de l'INSEE. 
TAB. 2 - Ratios de Remplacement à 60 ans

\begin{tabular}{c|cc}
\hline \hline & \multicolumn{2}{|c}{ Salaire Net } \\
en $\%$ & Modèle & Données \\
\hline & & \\
Cadres & 66 & 54 \\
Prof. Int. & 70 & 80 \\
Emp. Ouv. & 89 & 92 \\
& & \\
\hline \hline
\end{tabular}

\subsection{Une taxe particulièrement élevée dans le système par répartition français}

Avant d'illustrer les effets des surcotes actuariellement neutres, il est essentiel de mesurer la taxe sur la poursuite d'activité au delà du taux plein. Sur le graphique 3 sont reportés les ratios de remplacement issus du modèle étalonné pour le système de retraite actuel (160 trimestres de cotisation après la réforme Balladur). Le niveau des ratios de remplacement à 60 ans est proche de celui observé (tableau 2 - les ratios de remplacement observés sont extraits de [Colin et al., 2000]). Nous avons vérifié de façon générale l'adéquation du modèle aux données. Pour l'étalonnage de référence, le modèle parvient à reproduire certaines caractéristiques de l'économie française : taux de dépendance, compte des caisses de retraite (CNAV, ARRCO et AGIRC), taux de cotisation, comportements d'épargne et de départ à la retraite.

Le graphique 3 illustre la contrainte que les modalités du système de retraite fait peser sur les choix liés à la date de départ en retraite. Si les individus cessent leur activité avant d'avoir contribué 40 années au système de retraite (en moyenne aux âges de 63 ans, 60 ans et 58 ans pour les cadres, professions intermédiaires et employés - ouvriers respectivement), ils subissent une forte réduction de leur pension de retraite. En outre, une activité prolongée ne se traduit que par une faible augmentation du ratio de remplacement ${ }^{6}$. Le profil des ratios de remplacement présente donc un coude à l'âge du taux plein de chaque CSP.

Pour aller plus loin, il est nécessaire de calculer explicitement la taxe marginale associée à la poursuite d'activité, notée $\tau_{\text {marginal }}$ et définie par :

$$
\tau_{\text {marginal }}(z)=\frac{\left(\sum_{i=0}^{T(z)} \frac{P_{z}}{(1+r)^{i}}-\Pi_{z z+1} \sum_{i=1}^{T(z+1)} \frac{P_{z+1}}{(1+r)^{i}}\right)+\theta w(z)}{w(z)}
$$

avec $\tau_{\text {marginal }}(z)$ la taxe marginale associée à la poursuite d'activité d'un individu d'âge $z$, $P_{z+1}$ la pension de retraite en cas de cessation d'activité à l'âge $z+1, \Pi_{z z+1}$ la probabilité de survie entre les âges $z$ et $z+1, T(z)$ l'espérance de vie à l'âge $z, \theta$ le taux de cotisation, $w(z)$ le salaire à l'âge $z$ et $r$ le taux d'actualisation.

Nous retrouvons les deux éléments de la taxe sur la poursuite d'activité suggérés par le modèle théorique. En cas de départ retardé d'une année, la pension de retraite ne compense pas l'individu

- pour les cotisations supplémentaires payées pendant la poursuite d'activité

- pour le temps moindre pendant lequel l'individu jouit de sa retraite

\footnotetext{
${ }^{6}$ L'amélioration du ratio de remplacement en cas de départ au delà du taux plein résulte de l'augmentation du salaire des 10 meilleures années et de la contributivité des retraites complémentaires.
} 
FIG. 3 - Ratio de Remplacement après la réforme Balladur (160 trimestres de cotisation)

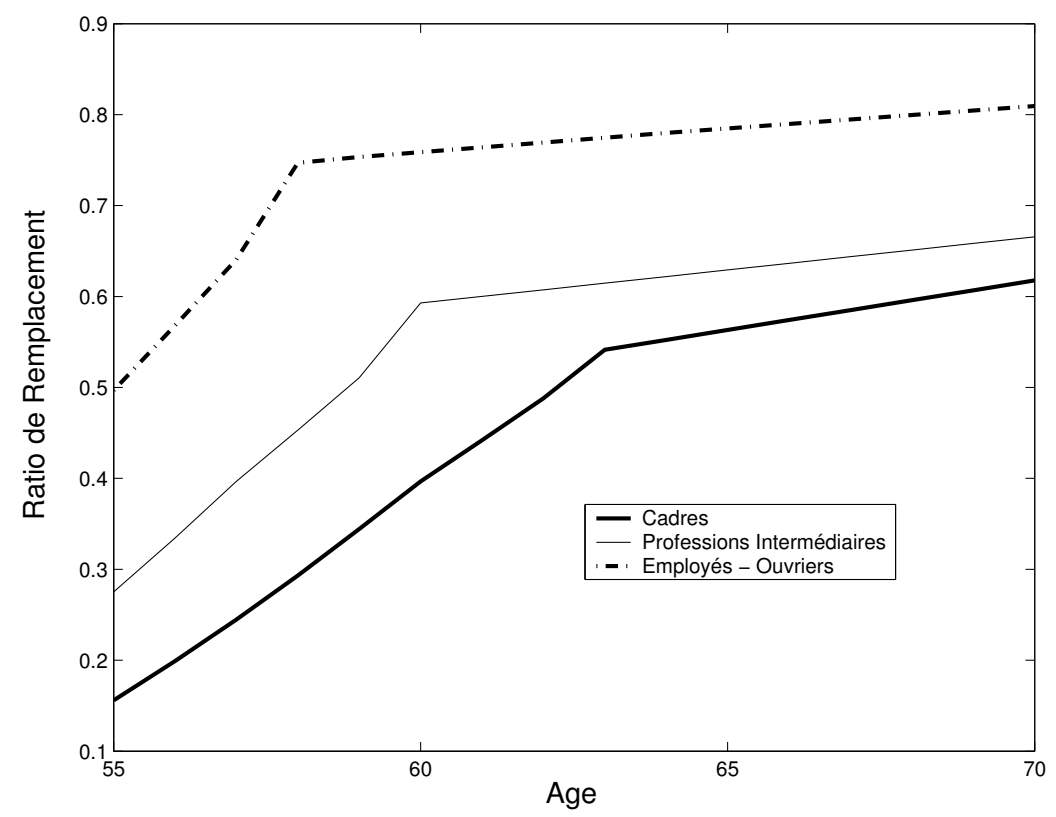

TAB. 3 - Taux marginal sur la poursuite d'activité(en \%)

\begin{tabular}{|c|c|c|c|c|c|c|c|c|c|c|}
\hline \multicolumn{11}{|c|}{ Régime Général CNAV } \\
\hline Age & 60 & 61 & 62 & 63 & 64 & 65 & 66 & 67 & 68 & 69 \\
\hline Cadres & 8,66 & 12,08 & 15,54 & 47,32 & 47,45 & 47,57 & 47,70 & 47,83 & 47,96 & 48,10 \\
\hline Professions Intermédiaires & 69,82 & 70,05 & 70,27 & 70,51 & 70,74 & 70,99 & 71,23 & 71,49 & 71,74 & 72,00 \\
\hline Employés Ouvriers & 93,92 & 94,32 & 94,74 & 95,16 & 95,98 & 96,36 & 96,74 & 97,13 & 97,53 & 97,94 \\
\hline \multicolumn{11}{|c|}{ Retraites Complémentaires ARRCO et AGIRC } \\
\hline Age & 60 & 61 & 62 & 63 & 64 & 65 & 66 & 67 & 68 & 69 \\
\hline Cadres & 13,41 & 15,78 & 18,36 & 39,16 & 41,11 & 43,07 & 45,06 & 47,08 & 49,12 & 51,19 \\
\hline Professions Intermédiaires & 21,89 & 23,12 & 24,37 & 25,63 & 26,91 & 28,21 & 29,53 & 30,86 & 32,22 & 33,59 \\
\hline Employés Ouvriers & 24,00 & 24,91 & 25,83 & 26,76 & 27,71 & 28,68 & 29,65 & 30,65 & 31,66 & 32,68 \\
\hline
\end{tabular}


L'étalonnage de référence ${ }^{7}$ conduit aux valeurs reportées dans le tableau 3. Dans le régime général, au delà de l'âge du taux plein, la taxe sur la poursuite d'activité est significative, en particulier pour les employés - ouvriers et les professions intermédiaires qui sont caractérisés par une espérance de vie moindre que celle des cadres. De plus, la taxe des régimes complémentaires est moindre que celle de la CNAV car cette partie de la pension de retraite est fondée sur un système plus contributif. Enfin, avant l'âge du taux plein, ce qui n'est effectif que pour les cadres dans nos simulations, la taxe est inférieure au taux de cotisation car la pension de retraite augmente significativement avec l'âge.

\subsection{Le niveau de déficit attendu}

L'espérance de vie va s'allonger progressivement pour atteindre selon les projections démographiques du [COR, 2001] une durée espérée de la période de retraite supérieure de 6 ans à celle que l'on connaît actuellement. Si l'on suppose une hétérogénéité identique, cela implique des espérances de vie à l'âge de 60 ans de 30,4 ans pour les cadres, de 26,7 ans pour les professions intermédiaires et de 24,6 pour les employés-ouvriers ${ }^{8}$. Comme nous l'avons souligné dans la section 2, l'allongement de l'espérance de vie conduit à un déficit des caisses de retraite si l'on laisse inchangé les paramètres du système par répartition. Le modèle conduit à une estimation d'un déficit tel que $29 \%$ des pensions ne sont pas financées à l'horizon de 2040 si aucune réforme n'est mise en place. Pour les caisses complémentaires, l'amplitude du déficit est similaire, car les taux de cotisation sont calculés sur la base de l'espérance de vie actuelle.

L'introduction de surcotes actuariellement neutres ne sauraient, par définition, remédier à ces déséquilibres financiers. Toutefois, prélever une fraction de ces surcotes contribuerait à financer le déficit futur tout en incitant les individus à prolonger leur activité. Comme nous l'avons souligné dans la section 2, une telle politique donne lieu à une courbe de Laffer dont le maximum $\tau^{L}$ est déterminé par simulation du modèle.

\subsection{Les surcotes actuariellement neutres}

Le niveau de la surcote actuariellement neutre détermine les potentialités d'un système incitatif. Plus elle est élevée, plus les caisses de retraite auront de marges pour prélever un surplus sur un niveau d'incitations suffisant pour retarder le départ en retraite, ie. à désutilité du travail donnée.

Rappelons que, puisqu'il s'agit de corriger les deséquilibres financiers futurs, nous ne modifions pas les décotes appliquées aujourd'hui en cas de départ en retraite avant l'âge du taux plein. Raisonnons sur un individu d'âge $z$ décidant de reporter son départ à la retraite à l'âge $z+1$, ce qui lui permettra de bénéficier d'une surcote, notée $\lambda_{2}^{*}(z+1)$. Il a déjà non seulement un taux plein, mais également une surcote résultant de ses choix précédents de report. La CNAV doit alors financer sur une période allant de l'âge $z+1$ à la fin de vie de l'agent une pension $\left(\lambda_{2}^{*}(z+1)+0.5\right) w^{\text {ref }}(z+1)$ où $w^{\text {ref }}(z+1)$ désigne le salaire de référence pour un âge $z+1$ de liquidation. Cependant, à l'âge $z$, cet individu paie à la

\footnotetext{
${ }^{7}$ Pour l'étalonnage de référence, l'espérance de vie est celle observée aujourd'hui, le taux d'actualisation est le taux d'intérêt auquel ont accès les ménages sur les marchés financiers, le taux de cotisation est celui obtenu à l'équilibre des caisses de retraite pour un barème postérieur à la réforme Balladur et le salaire est étalonné sur données françaises. Une description détaillée de l'étalonnage est fournie dans [Hairault et al., 2004].

${ }^{8}$ On pourrait supposer une certaine convergence de ces espérance de vie. Nous avons cependant préféré raisonner à hétérogénéité constante.
} 
TAB. 4 - Surcotes Actuariellement Neutres

\begin{tabular}{lccccccccc}
\hline \hline$\tilde{r}=0 \%$ & \multicolumn{8}{c}{$\lambda_{2}^{*}(z+1)-\lambda_{2}^{*}(z)$} \\
\hline Age $z+1$ & 61 & 62 & 63 & 64 & 65 & 66 & 67 & 68 & 69 \\
\hline Cadres & 0 & 0 & 3,35 & 3,61 & 3,92 & 4,26 & 4,63 & 5,04 & 5,51 \\
Professions Int. & 3,58 & 3,9 & 4,26 & 4,66 & 5,12 & 5,62 & 6,2 & 6,86 & 7,61 \\
Employés-ouvriers & 3,75 & 4,12 & 4,52 & 5,04 & 5,58 & 6,2 & 6,9 & 7,72 & 8,66 \\
\hline \hline
\end{tabular}

CNAV une cotisation égale à $\tau w(z)$. En outre, la CNAV lui aurait versé une pension de $\left(\lambda_{2}^{*}(z)+0.5\right) w^{r e f}(z)$ si l'agent était parti à la retraite à l'âge $z$. Financièrement, la CNAV est indifférente entre ces deux options si la surcote vérifie :

$$
\Pi_{z z+1} \sum_{i=1}^{T(z+1)} \frac{\left(\lambda_{2}^{*}(z+1)+0.5\right) w^{r e f}(z+1)}{(1+\tilde{r})^{i}}-\theta w(z)=\sum_{i=0}^{T(z)} \frac{\left(\lambda_{2}^{*}(z)+0.5\right) w^{r e f}(z)}{(1+\tilde{r})^{i}}
$$

Le membre de droite représente les gains pour la CNAV de cette stratégie : économie des pensions versées si l'individu était parti à l'âge $z$ et les cotisations reçues en raison de la décision de reporter d'une année le départ à la retraite.

Comme la probabilité de mort est croissante avec l'âge, le niveau des surcotes marginales (entre deux âges successifs, $\left.\lambda_{2}^{*}(z+1)-\lambda_{2}^{*}(z)\right)$ est lui-même croissant et n'est donc pas constant comme cela est proposé dans la réforme du gouvernement Raffarin (surcote de $3 \%$ ). Ce point est important dans la mesure où cette croissance doit compenser la décroissance de l'espérance de vie. Le profil des surcotes marginales doit donc être croissant pour être le plus efficace.

La valeur du rendement $\tilde{r}$ apparaissant dans l'expression (19) est cruciale dans le niveau des surcotes. Dans un régime par répartition, dans lequel les surplus des caisses sont répartis aux différentes générations de retraités, ce rendement est implicite et découle de la croissance démographique, et potentiellement du rythme du progrès technique s'il n'y a pas parfaite indexation des pensions sur les salaires. Par exemple, en régime stationnaire, le report d'un an par une génération peut permettre de financer, toutes choses égales par ailleurs, des retraites plus généreuses pour les générations précédentes de retraités ayant opéré le même choix de report, parce que ces dernières sont moins nombreuses du fait de la croissance démographique. Dans ce cas, le rendement $\tilde{r}$ provient du régime par répartition même. Sous l'hypothèse d'absence de croissance démographique, le rendement $\tilde{r}$ est égal à zéro ${ }^{9}$. Dans le tableau 4 sont reportés les accroissements de surcotes $\lambda_{2}^{*}(z+1)-\lambda_{2}^{*}(z)$ pour des valeurs croissantes de l'âge.

Deux facteurs expliquent les niveaux des majorations reportées dans le tableau 4.

- Plus l'espérance de vie est faible au moment du départ en retraite, plus les surcotes sont élévées, puisque la pension majorée doit être versée moins longtemps. Ce facteur explique des niveaux plus faibles pour les cadres, mais surtout une croissance

\footnotetext{
${ }^{9}$ Toutefois, les surplus générés par le recul de l'âge de retraite pourraient être placés par les caisses de retraite sur les marchés financiers afin de profiter à la marge du rendement de la capitalisation. Dans ce cas, $\tilde{r}$ correspond à un taux d'intérêt de marché. Cela suppose une gestion financière des économies procurées par le report de l'âge de la retraite au-delà du taux plein. Nous mesurons dans [Hairault et al., 2004] les implications d'hypothèses alternatives sur le taux de rendement $\tilde{r}$.
} 
FIG. 4 - Ratio de remplacement avec les surcotes actuariellement neutres

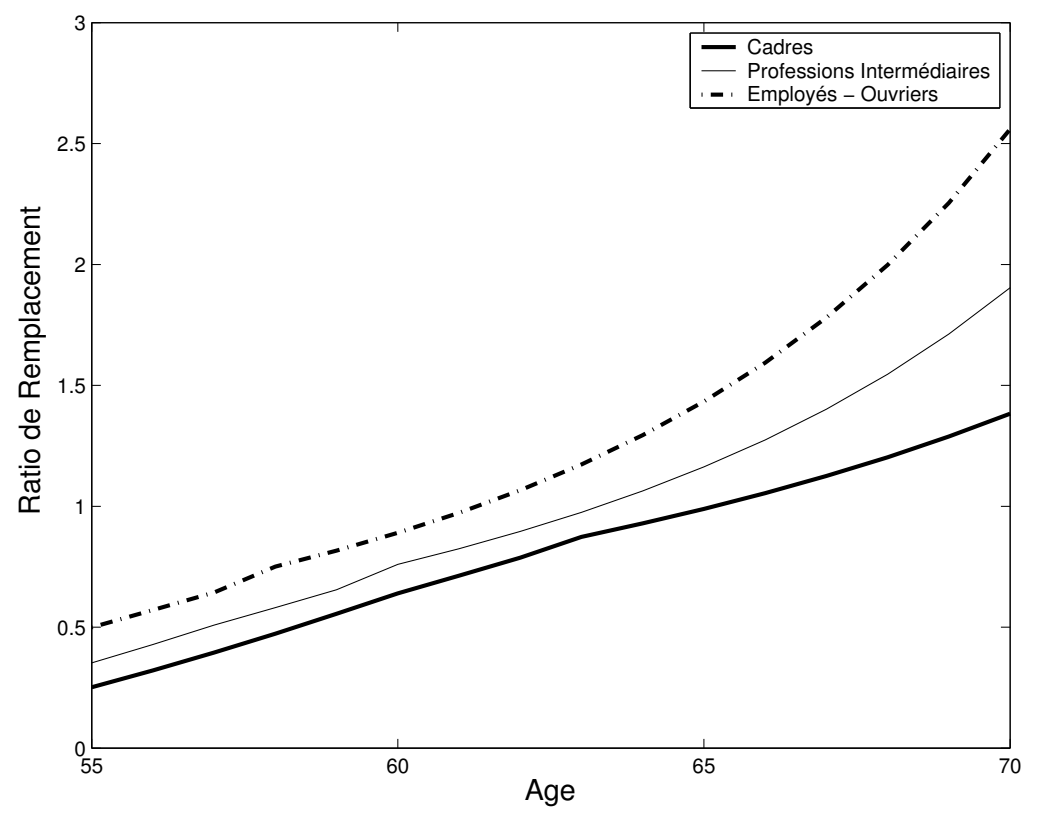

des surcotes par rapport à l'âge, quelle que soit la CSP considérée. Ces majorations marginales croissantes se cumulent puisque les actifs ont acquis les années précédentes des majorations qu'il faut dépasser pour les inciter à travailler encore plus longtemps.

- Un second élément important tient à la modification du salaire de référence induite par une année d'activité supplémentaire. Les surcotes des ouvriers sont minorées par cet élément, toutes choses égales par ailleurs, car toute année active supplémentaire fait augmenter le salaire de référence, à la différence des autres catégories qui ont des salaires de fin de vie active au-delà du plafond de la sécurité sociale.

Le graphique 4 présente les ratios de remplacement lorsque les individus reçoivent la surcote actuariellement neutre en cas de départ retardé. Après la réforme Balladur, rappelons qu'en moyenne l'âge du taux plein est atteint à 63,60 et 58 ans pour les cadres, les professions intermédiaires et les employés - ouvriers respectivement. Contrairement au graphique 3, les individus bénéficient d'un ratio de remplacement croissant au fur et à mesure qu'ils poursuivent leur activité. En effet, le surplus de pension en cas de départ retardé compense l'individu pour le moindre temps passé en retraite lorsqu'il diffère sa cessation d'activité.

\subsection{Le sommet de la courbe de Laffer}

Comme l'a montré simplement la section analytique, la détermination du sommet de la courbe de Laffer dépend fondamentalement du paramètre relatif à l'utilité du loisir. Compte tenu de la taxe élevée sur la prolongation d'activité existant actuellement, il est difficile de révéler ce paramètre à partir des âges de départ observés. Le départ à l'âge du taux plein informe uniquement sur un niveau minimal de l'utilité du loisir en dessous duquel certains agents retarderaient leur départ. Ce niveau du paramètre déterminant l'utilité 
du loisir fixe d'une certaine façon la borne maximale d'efficacité d'une réforme visant à introduire des incitations à la prolongation d'activité. Nous déterminons par simulation ce paramètre. Si l'observation des départs en retraite n'apporte pas d'informations suffisantes pour fonder un étalonnage des paramètres caractérisant les préférences des agents, il est possible de se caler sur le niveau de la consommation dans le PIB.

Considérons la fonction d'utilité instantanée suivante :

$$
u(c, l)=\frac{\left(c^{1-\eta}(1-l)^{\eta}\right)^{1-\tilde{\sigma}}}{1-\tilde{\sigma}}
$$

$c$ symbolisant la consommation (annuelle), $l$ le temps passé à travailler. Si l'on définit une transformation de la fonction d'utilité des agents comme suit :

$$
\frac{c^{1-\sigma}}{1-\sigma} \vartheta(l)=\frac{\left(c^{1-\eta}(1-l)^{\eta}\right)^{1-\tilde{\sigma}}}{1-\tilde{\sigma}}
$$

alors, par identification terme à terme, l'exposant de la consommation est $(1-\eta)(1-\tilde{\sigma})=$ $1-\sigma$. Traditionnellement, le paramètre $\sigma$ à est fixé à une valeur de 2. Cet étalonnage correspond en particulier à l'estimation sur données de panel de [Attanasio et al., 1999]. Il n'existe pas d'estimation comparable sur données françaises. On suppose par ailleurs que la durée quotidienne de travail est de 8 heures pour les employés qui disposent d'une dotation en temps de 24 heures, d'où $1-l=2 / 3$. Pour les chômeurs, les pré-retraités et les retraités, on a $l=0$. Suivant [Hansen $\&$ Imrogoroglu, 1992], on peut donc en déduire le paramètre $\eta$ de l'évaluation du taux marginal de substitution entre consommation et loisir à l'état stationnaire :

$$
\frac{\eta}{1-\eta} \frac{c}{1-l}=w \Rightarrow \eta=\left[1+\left(\frac{w l / y}{c / y}\right)\left(\frac{1-l}{l}\right)\right]^{-1}
$$

Ceci permet alors d'en inférer la valeur traditionnellement retenue de $\eta=0.67$, pour une part de la consommation des biens non-durables dans le PIB $(y)$ égale à 0.69 , compte tenu d'une part des salaires égale à 0.70 en France. On obtient dans ce cas une valeur de $\widetilde{\sigma}=4.03$ par identification. 0.67 est considérée comme la valeur macroéconomique de $\eta$, tandis que sa valeur minimale, obtenue par simulation selon le principe décrit précédemment, pour un étalonnage correspondant à la réforme Balladur et une espérance de vie observée aujourd'hui, est de 0.60. Rappelons qu'en dessous de cette valeur notre modélisation conduit certains agents à partir avant le taux plein. Il s'agit donc bien de la valeur minimale que peut prendre ce paramètre pour rester compatible avec l'observation ${ }^{10}$. Dans ce cas, $\widetilde{\sigma}$ est égal à 3.50 .

Le tableau 5 fournit une approximation de la courbe de Laffer en France pour ces deux valeurs de $\eta$. Quelle que soit cette valeur, nous retrouvons les deux points extrêmes de la courbe de Laffer :

- Lorsque la CNAV taxe peu les incitations actuariellement neutres ( $\tau$ faible), les individus reçoivent de fortes incitations à différer leur départ en retraite. Toutefois, par définition, cette stratégie ne contribue guère à la correction des déséquilibres financiers, malgré des ratios de dépendance fortement abaissés.

\footnotetext{
${ }^{10}$ Dans le cadre de notre modélisation qui ne concerne que les carrières complètes, cela produit une distribution des âges de départ en retraite caractérisée par deux pics : un pic à 63 ans pour les cadres et un pic à 60 ans pour les deux autres CSP, correspondant aux âges de taux plein de chaque CSP sous le régime Balladur.
} 
- De même, lorsque la CNAV souhaite prélever une forte proportion de la surcote actuariellement neutre ( $\tau$ élevé), les individus ne bénéficient que d'une faible augmentation de leur pension en cas d'activité prolongée, ce qui limite la baisse du ratio de dépendance et ainsi les possibilités de financement.

On constate que la réduction maximale du déficit de la CNAV est égale à $8.6 \%$ pour une utilité du loisir calibrée de façon macroéconomique. Elle serait au maximum égale à $30.12 \%$ pour un niveau minimal de l'utilité du loisir. Dans ce dernier cas, la CNAV peut se permettre de donner des surcotes moins élevées, en prélevant à un taux plus élevé $(0.4$ contre 0.3 ), tout en retardant avec succès le départ à la retraite. Au sommet de la courbe de Laffer, la CNAV prélève entre $40 \%$ et $30 \%$ des surcotes actuariellement neutres, ce qui lui permet de financer une partie de ses besoins de financement futurs.

TAB. 5 - Incidence des incitations

\begin{tabular}{c|cccc}
\hline \hline & \multicolumn{3}{|c}{ Deficit } & \multicolumn{2}{c}{ Dépendance } \\
$\tau$ & $\eta=0.67$ & $\eta=0.60$ & $\eta=0.67$ & $\eta=0.60$ \\
& & & & \\
\hline 0 & 0 & 0 & 51.3 & 45.3 \\
0.2 & -6.7 & -20.64 & 53.3 & 45.5 \\
0.3 & -8.6 & -25.49 & 53.8 & 47.6 \\
0.4 & -6.9 & -30.12 & 54.9 & 48.2 \\
0.5 & -5.4 & -29.66 & 55.5 & 50.6 \\
0.6 & -3.0 & -25.51 & 56.1 & 52.4 \\
\hline \hline
\end{tabular}

Les valeurs des colonnes "Déficit" correspondent aux variations des déficits des caisses rapportées à leurs déficits respectifs de référence.

\section{Conclusion}

Partant d'une situation où la prolongation d'activité au-delà du taux plein dans le Régime Général est fortement taxée, nous proposons la mise en place de bonus ou de surcotes qui permettraient à la fois de se rapprocher de la neutralité actuarielle et de financer en partie les déficits futurs. Une telle stratégie, en réduisant la taxe sur la poursuite d'activité, incite les individus à retarder leur départ en retraite, contribuant ainsi à la réduction des besoins de financement. Le choix de la fraction de la surcote actuariellement neutre accordée aux individus donne naissance à une courbe de Laffer : un arbitrage s'opère entre accorder davantage d'incitations financières aux individus désireux de retarder leur départ en retraite, ce qui limite la réduction du déficit, et proposer des surcotes moins généreuses qui n'incitent guère les individus à poursuivre leur activité. Notre article fournit une approximation de cette courbe de Laffer sur données françaises. Au sommet de la courbe de Laffer, la CNAV prelève entre 30 et $40 \%$ des surcotes actuariellement neutres, ce qui lui permet de financer une partie de ses besoins futurs. Ce résultat donne un certain crédit à la surcote de $3 \%$ octroyée par le gouvernement Raffarin.

Nos résultats sont bien entendu sensibles aux hypothèses retenues concernant les transitions sur le marché du travail. Notre évaluation est effectuée sous un scénario favorable 
pour les travailleurs âgés. Dans le modèle, ces derniers sont certains de conserver leur emploi après 60 ans. Or, l'employabilité des travailleurs âgés constitue l'une des grandes interrogations des réformes des retraites, interrogation qui n'est pas intégrée dans cet article. Nous avons vérifié que, conformément à l'intuition, le sommet de la courbe de Laffer conduit à une moindre réduction des déficits que celle obtenue dans cet article lorque l'on prend en compte un risque de retraite contrainte : certains agents partent dès leur taux plein parce qu'ils ne sont plus assurés de pouvoir différer longtemps leur départ. Toutefois, notre calibration de base a retenu un taux de chômage en fin de vie active tel qu'il est observé actuellement, ie. près de la moitié de la population active n'est pas en mesure de prolonger son activité, ce qui diminue fortement l'efficacité de la réforme proposée. Au total, compte tenu des incertitudes sur l'évolution du taux d'activité des vieux actifs, il est difficile d'aller plus loin dans cette analyse, mais il est évident qu'il existe une forte complémentarité, certainement dans les deux sens, entre une politique d'incitation à la prolongation d'activité et un taux d'emploi plus élévé des travailleurs âgés.

Une seconde dimension négligée dans notre présente analyse mériterait d'être approfondie. Elle a trait à la forte hétérogénéité de l'état de santé parmi les travailleurs âgés. La disparité des espérances de vie pourrait conduire à un phénomène de sélection adverse : seuls ceux qui se considèrent en bonne santé accepteraient de retarder leur départ en retraite. Parce que la surcote actuariellement neutre est calculée sur l'espérance de vie moyenne, le déficit des caisses de retraite pourrait alors se creuser, et non partiellement se résorber. [Cremer et al., 2004] soulignent par ailleurs, qu'en présence d'asymétrie d'information sur l'état de santé et la productivité des individus, l'optimum social se situe sur la partie croissante de la courbe de Laffer. Dans cette étude, nous avons utilisé une information objective sur les différences d'espérance de vie entre les catégories socio-professionnelles pour calculer des surcotes propres à chaque catégorie. Au-delà des difficultés que poseraient déjà cette différenciation à l'interieur du système par répartition, demeure un délicat problème d'information imparfaite à l'intérieur de chaque CSP. De ce point de vue, les surcotes proposées aux travailleurs âgés devraient permettre de traiter de façon pertinente la présence en information asymétrique de mauvais risques (ceux dont l'espèrance de vie est élevée) et les bons risques (ceux dont l'éspérance de vie est faible). Une façon d'éviter ce problème d'information imparfaite serait de permettre pour la partie majorée de la pension une sortie en capital, calculée sur les sommes effectivement économisées par les caisses de retraite qui ne feraient donc pas intervenir l'espérance de vie. Le montant de ce capital apparaîtrait d'ailleurs peut-être plus incitatif que la majoration marginale d'une rente.

\section{Références}

[Artus \& Legros, 1999] Artus, P. \& Legros, F. (1999). Le Choix Du Système de Retraite. Economica.

[Attanasio et al., 1999] Attanasio, O. P., Banks, J., Meghir, C., \& Weber, G. (1999). Humps and bumps in lifetime consumption. Journal of Business and Economic Statistics, 17.

[Bardaji et al., 2002] Bardaji, J., Sédillot, B., \& Walraet, E. (2002). Evaluation de Trois Réformes Du Régime Général D'assurance Vieillesse À L'aide Du Modèle de Microsimulation DESTINIE. Documents de travail de la Direction des Etudes et Synthèses Economiques G 2002 / 07, INSEE. 
[Blanchard, 1985] Blanchard, O. (1985). Debt, deficits and finite horizons. Journal of Political Economy, 93, 223-247.

[Blanchet \& Mahieu, 2001] Blanchet, D. \& Mahieu, R. (2001). Une analyse microéconométrique des comportements de retrait d'activité. Revue d'Economie Politique, numéro spécial Epargne et Retraite, 9-31.

[Castañeda et al., 2002] Castañeda, A., Diaz-Gimenez, J., \& Rios-Rull, V. (2002). Accounting for the u.s. earnings and wealth inequality. Journal of Political Economy.

[Colin et al., 2000] Colin, C., Iéhlé, V., \& Mahieu, R. (2000). Les trajectoires de fin de carrière des salariés du secteur privé. Solidarité-Santé, (3).

[COR, 2001] COR (2001). Retraites : Renouveler Le Contrat Social Entre Les Générations. Conseil d'Orientation des Retraites.

[Cremer et al., 2004] Cremer, H., Lozachmeur, J. M., \& Pestieau, P. (2004). Social security, retirement age and optimal income taxation. Journal of Public Economics, 88(11), $2259-2281$.

[D'Autume, 2003] D'Autume, A. (2003). Vieillissement et choix de l'âge de la retraite. que peut nous dire le modèle à générations? Revue Economique, 54(3), 561-572.

[Desmet \& Pestieau, 2003] Desmet, R. \& Pestieau, P. (2003). Sécurité sociale et départ à la retraite. Revue Française d'Economie, XVIII(2).

[Gertler, 1999] Gertler, M. (1999). Government debt and social security in a life-cycle economy. Carnegie Rochester Conference Series on Public Policy, 50, 61-110.

[Gruber \& Wise, 1998] Gruber, J. \& Wise, D. (1998). Social security and retirement : An international comparison. American Economic Review, 88(2), 158-163.

[Hairault \& Langot, 2002] Hairault, J. \& Langot, F. (2002). Inégalités et réformes des retraites. Revue Française d'Economie, XVII, 97-159.

[Hairault et al., 2004] Hairault, J., Langot, F., \& Sopraseuth, T. (2004). Retraite choisie et réduction des déficits. Revue Française d'Economie, XVIII(3), 75-136.

[Hansen \& Imrogoroglu, 1992] Hansen, G. \& Imrogoroglu, A. (1992). The role of unemplyment insurance in an economy with liquidity constraints and moral hazard. Journal of Political Economy, 100, 118-142.

[INGENUE, 2001] INGENUE, E. (2001). Vieillissement démographique et transferts internationaux d'épargne. Revue d'Economie Politique, Numéro Spécial Epargne et Retraite, $195-228$.

[Mahieu \& Sédillot, 2001] Mahieu, R. \& Sédillot, B. (2001). Départ à la retraite, irréversibilité et incertitude. Economie et Prévision, 2001-3(149), 87-101.

[Rust \& Phelan, 1997] Rust, J. \& Phelan, C. (1997). How social security and medicare affect retirement behavior in a world of incomplete markets. Econometrica, 65(4), 781831.

[Yaari, 1965] Yaari, M. F. (1965). Uncertain lifetime, life insurance, and the theory of consumer. Review of Economic Studies, 32, 137-158. 